\title{
Identification of Quantitative Trait Loci That Determine Plasma Total-Cholesterol and Triglyceride Concentrations in DDD/Sgn and C57BL/6J Inbred Mice
}

\author{
Jun-ichi Suto ${ }^{1}$ and Misaki Kojima ${ }^{2}$ \\ ${ }^{1}$ Institute of Agrobiological Sciences, National Agriculture and Food Research Organization (NARO), Tsukuba, \\ Ibaraki 305-8634, Japan \\ ${ }^{2}$ Institute of Livestock and Grassland Science, National Agriculture and Food Research Organization (NARO), \\ Tsukuba, Ibaraki 305-0901, Japan \\ Correspondence should be addressed to Jun-ichi Suto; jsuto@affrc.go.jp
}

Received 28 November 2016; Revised 13 April 2017; Accepted 11 May 2017; Published 31 May 2017

Academic Editor: Daniel Gaudet

Copyright (C) 2017 Jun-ichi Suto and Misaki Kojima. This is an open access article distributed under the Creative Commons Attribution License, which permits unrestricted use, distribution, and reproduction in any medium, provided the original work is properly cited.

\begin{abstract}
$\mathrm{DDD} /$ Sgn mice have significantly higher plasma lipid concentrations than C57BL/6J mice. In the present study, we performed quantitative trait loci (QTL) mapping for plasma total-cholesterol $(\mathrm{CHO})$ and triglyceride $(\mathrm{TG})$ concentrations in reciprocal $\mathrm{F}_{2}$ male intercross populations between the two strains. By single-QTL scans, we identified four significant QTL on chromosomes (Chrs) 1, 5, 17, and 19 for CHO and two significant QTL on Chrs 1 and 12 for TG. By including cross direction as an interactive covariate, we identified separate significant QTL on Chr 17 for CHO but none for TG. When the large phenotypic effect of QTL on Chr 1 was controlled by composite interval mapping, we identified three additional significant QTL on Chrs 3, 4, and 9 for CHO but none for TG. QTL on Chr 19 was a novel QTL for CHO and the allelic effect of this QTL significantly differed between males and females. Whole-exome sequence analysis in DDD/Sgn mice suggested that Apoa2 and Acads were the plausible candidate genes underlying CHO QTL on Chrs 1 and 5, respectively. Thus, we identified a multifactorial basis for plasma lipid concentrations in male mice. These findings will provide insight into the genetic mechanisms of plasma lipid metabolism.
\end{abstract}

\section{Introduction}

Plasma lipid concentrations are representative quantitative traits; that is, they are controlled by multiple genes under the influence of nonheritable environmental effects. Among plasma lipids, cholesterol (CHO) and triglyceride (TG) have clinical implications in atherosclerosis and coronary artery disease; therefore, it is crucially important to identify genes influencing variations in plasma $\mathrm{CHO}$ and $\mathrm{TG}$ concentrations $[1,2]$ (in this manuscript, we use the term "plasma lipid" when $\mathrm{CHO}$ and TG are simultaneously referred to). Many studies have aimed to dissect the genetic bases underlying plasma lipid concentrations in mice, and more than one hundred CHO QTL and more than fifty TG QTL have been reported to date $([3,4]$ and Mouse Genome Informatics (MGI, http://www.informatics.jax.org/)). Most QTL identified in mice have conserved synteny with human loci that control plasma lipid concentrations and diseases [5].

We previously performed QTL mapping studies to identify plasma lipid concentrations using mouse intercrosses [69]. We identified several significant QTL for plasma $\mathrm{CHO}$ and TG concentrations in C57BL/6J $\times$ DDD.Cg- $A^{y} \mathrm{~F}_{2}$ female mice [9]. In the present study, we performed QTL mapping for plasma $\mathrm{CHO}$ and $\mathrm{TG}$ concentrations in $\mathrm{F}_{2}$ male mice produced via crosses between C57BL/6J and DDD/Sgn mice. Because male intercross population produced by these strains is not previously evaluated for plasma lipid concentrations, we expect novel QTL in addition to known QTL for plasma lipid concentrations to be identified. Furthermore, because there were apparent sex differences in plasma lipid concentrations between these mouse strains (e.g., female DDD.Cg$A^{y}$ mice had higher TG concentrations than males, whereas 
male C57BL/6J mice had higher TG concentrations than females [9]), we expect genetic aspects of sex differences to be revealed. Because men have a higher risk of coronary artery disease than women [10], it is crucially important to unravel the molecular basis of sex differences in plasma lipid concentrations.

\section{Materials and Methods}

2.1. Mice. The inbred mouse strains DDD and B6 were maintained at the National Institute of Agrobiological Sciences (NIAS, Tsukuba, Japan). Reciprocal crosses between DDD and $\mathrm{B} 6$ strains produced $\mathrm{DB}\left(\right.$ ODDD $\left.\times{ }_{0}^{\top} \mathrm{B} 6\right) \mathrm{F}_{1}$ and $\mathrm{BD}$ $\left(\right.$ oB $\left.6 \times{ }^{\top} \mathrm{DDD}\right) \mathrm{F}_{1}$ mice, both of which were intercrossed to produce $\mathrm{DB} \mathrm{F}_{2}(n=150)$ and $\mathrm{BD} \mathrm{F}_{2}(n=150)$ male mice [11].

All mice were weaned at 4 weeks of age and 4-5 mice were housed together in a cage during the experiments. All mice were maintained in a specific pathogen-free facility with a regular light cycle and controlled temperature and humidity. Food (CRF-1; Oriental Yeast Co., Ltd., Tokyo, Japan) and water were freely available throughout the experimental period. All animal procedures were reviewed and approved by the Institutional Animal Care and Use Committee of NIAS.

2.2. Plasma Lipid Analysis. Plasma lipid concentrations were determined at the age of 11 to 14 weeks in DDD, B6, and $\mathrm{F}_{1}$ mice and at the age of 11 to 12 weeks (71-80 days after birth) in $\mathrm{F}_{2}$ mice.

Mice were euthanized with an overdose of ether immediately after weighing in the morning. Blood was collected from the heart of an individual mouse using heparin as an anticoagulant. Plasma was separated by centrifugation at $2,000 \times \mathrm{g}$ for $15 \mathrm{~min}$ at $4^{\circ} \mathrm{C}$ and was stored at $-80^{\circ} \mathrm{C}$ until use. Plasma $\mathrm{CHO}$ and $\mathrm{TG}$ concentrations were determined by enzymatic methods using clinical chemical kits (Wako Pure Chemical Industries, Osaka, Japan).

2.3. Genotyping. Microsatellite sequence length polymorphisms were identified by electrophoresis after PCR amplification of genomic DNA. PCR amplification was carried out by use of a TaKaRa PCR Thermal Cycler Dice (TaKaRa Bio Inc., Shiga, Japan) under the following conditions: 1 cycle at $94^{\circ} \mathrm{C}$ for $3 \mathrm{~min} ; 35$ cycles at $94^{\circ} \mathrm{C}$ for $30 \mathrm{~s}, 55^{\circ} \mathrm{C}$ for $1 \mathrm{~min}$, and $72^{\circ} \mathrm{C}$ for $45 \mathrm{~s} ; 1 \mathrm{cycle}$ at $72^{\circ} \mathrm{C}$ for $7 \mathrm{~min}$. PCR products were separated on $10 \%$ polyacrylamide gel (Nacalai Tesque Inc., Kyoto, Japan) and were visualized by ethidium bromide (Nacalai Tesque) staining. A total of 117 microsatellite loci were genotyped. Their chromosomal positions were retrieved from MGI.

2.4. QTL Analysis. Normality of the trait data was assessed by Shapiro-Wilk $W$ test (JMP8, SAS Institute Japan Inc., Tokyo, Japan). If the trait data did not follow a normal distribution, Box-Cox transformation was applied to the raw trait data (JMP8).

QTL mapping was performed using R/qtl version 1.38$4[18,19]$. Single-QTL scans were performed by computing at $1 \mathrm{cM}$ intervals across the entire genome using the cross direction (DB versus BD) as a covariate. Threshold logarithm of the odds (LOD) scores for significant $(P<0.05)$ and suggestive $(P<0.63)$ linkages were determined by performing 1,000 permutations [20]. After single-QTL scans, two-QTL scans were performed. In this case, we adhered strictly to the threshold recommended by Broman and Sen [18]. Finally, the combined effects of covariates and all QTL_including those that were significant and suggestive-were assessed using multiple QTL models [21].

2.5. Whole-Exome Sequence Analysis. Genomic DNA was extracted from the tail of DDD mice using a genomic DNA purification kit (Wizard Genomic DNA Purification Kit, Promega KK, Tokyo, Japan) and was submitted to Filgen, Inc. (Nagoya, Aichi, Japan) for exome capture and sequencing. Briefly, genomic DNA was subjected to the agarose gel and $\mathrm{OD}$ ratio tests to confirm the purity and concentration prior to Bioruptor (Diagenode, Inc., Denville, NJ, USA) fragmentation. Fragmented genomic DNAs were tested for size distribution and concentration using an Agilent Bioanalyzer 2100 and Nanodrop (Agilent Technologies, Wilmington, DE, USA). Illumina libraries were made from qualified fragmented genomic DNA using SPRIworks HT reagent kit (Beckman Coulter, Inc., Indianapolis, IN, USA), and the resulting libraries were subjected to exome enrichment using SureSelect XT Mouse All Exon Kit (Agilent Technologies) following the manufacturer's instructions. Enriched libraries were tested for enrichment by qPCR and for size distribution and concentration by an Agilent Bioanalyzer 2100. The samples were then sequenced on an Illumina HiSeq2000 (Illumina, San Diego, CA, USA), which generated pairedend reads of 90 or 100 nucleotides. Data was analyzed for data quality using FASTQC (Babraham Institute, Cambridge, $\mathrm{UK})$. Sequence reads were mapped to the mouse reference genome (GRCm38, mm10). Read mapping and variant analyses were performed using CLC Genomics Workbench 7.0.4 and 8.5.1 (Filgen).

2.6. Statistical Analysis. Plasma lipid concentrations are represented as the mean \pm SEM (mg/dL). Statistical differences between two groups were analyzed using Student's or Welch's $t$-tests. Tukey-Kramer honestly significant difference tests were used for statistical comparisons among more than two groups. $P$ values $<0.05$ were considered statistically significant.

\section{Results}

3.1. Plasma Lipid Concentrations in DDD, B6, and $F_{1}$ Mice. Table 1 shows the statistical comparison of plasma CHO and TG concentrations between DDD and B6 mice and between DB $F_{1}$ and $B D F_{1}$ mice. Parental and $F_{1}$ mice were analyzed separately. Among parental mice, both male and female DDD mice had significantly higher plasma lipid concentrations than their $\mathrm{B} 6$ counterparts. In $\mathrm{F}_{1}$ mice, excluding $\mathrm{CHO}$ concentrations in $\mathrm{DB} \mathrm{F}_{1}$ and $\mathrm{BD} \mathrm{F}_{1}$ males, both male and female $\mathrm{DB} \mathrm{F}_{1}$ mice had significantly higher plasma lipid concentrations than their $\mathrm{BD} \mathrm{F}_{1}$ counterparts. A clear lineage effect was thus observed. 
TABLE 1: Plasma lipid concentrations in $\mathrm{DDD}, \mathrm{B} 6, \mathrm{DB} \mathrm{F}_{1}$, and $\mathrm{BD} \mathrm{F}_{1}$ mice.

\begin{tabular}{|c|c|c|c|c|c|c|c|}
\hline \multirow{2}{*}{ Plasma lipids } & \multirow{2}{*}{ Sex } & \multicolumn{2}{|c|}{ Mean \pm SEM plasma lipids (mg/dL) } & \multirow{2}{*}{$P$ value } & \multicolumn{2}{|c|}{ Mean \pm SEM plasma lipids $(\mathrm{mg} / \mathrm{dL})(n)$} & \multirow{2}{*}{$P$ value } \\
\hline & & DDD & B6 & & $\mathrm{DB} \mathrm{F}_{1}$ & $\mathrm{BD} \mathrm{F}_{1}$ & \\
\hline \multirow{2}{*}{$\mathrm{CHO}$} & Males & $173 \pm 4(n=38)$ & $102 \pm 5(n=21)$ & $<0.0001$ & $137 \pm 4(n=15)$ & $128 \pm 5(n=12)$ & NS \\
\hline & Females & $162 \pm 3(n=31)$ & $92 \pm 3(n=21)$ & $<0.0001$ & $119 \pm 3(n=17)$ & $110 \pm 3(n=12)$ & $<0.05$ \\
\hline \multirow{2}{*}{ TG } & Males & $154 \pm 11(n=38)$ & $109 \pm 15(n=21)$ & $<0.03$ & $200 \pm 15(n=15)$ & $112 \pm 16(n=12)$ & $<0.0005$ \\
\hline & Females & $194 \pm 10(n=31)$ & $43 \pm 12(n=21)$ & $<0.0001$ & $108 \pm 9(n=17)$ & $65 \pm 10(n=12)$ & $<0.005$ \\
\hline
\end{tabular}

NS, not significant.

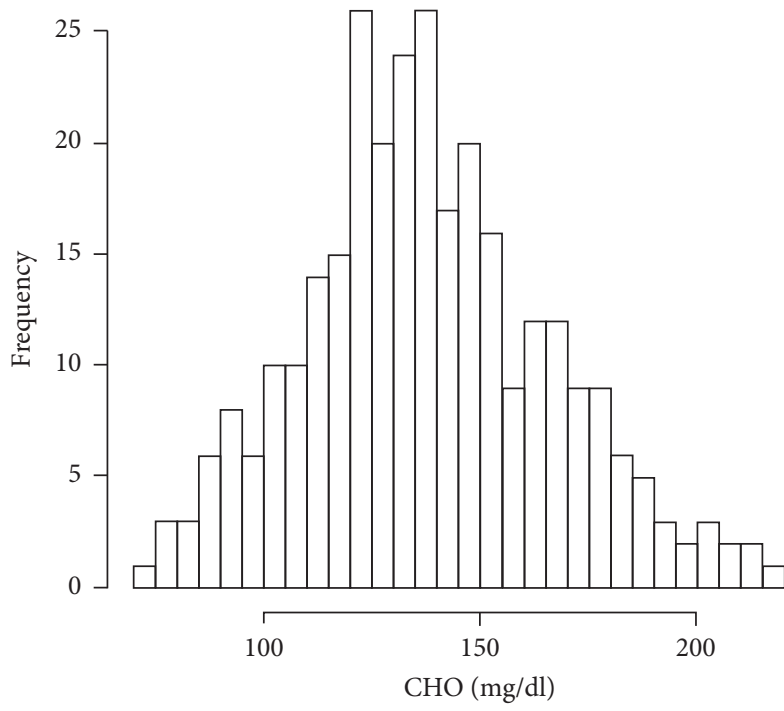

(a)

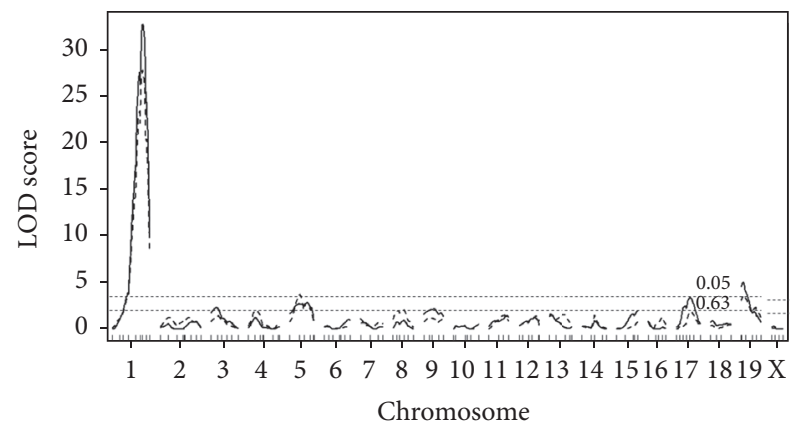

(b)

FIGURE 1: Genome-wide scan for plasma $\mathrm{CHO}$ concentrations in $\mathrm{F}_{2}$ male mice. (a) A histogram showing the distribution of plasma $\mathrm{CHO}$ concentrations. (b) Genome-wide LOD score plot of single-QTL scans for plasma CHO concentrations (solid lines: cross direction as an additive covariate; broken lines: cross direction and body weight as additive covariates). The $x$-axis represents the Chrs and microsatellite marker positions, and the $y$-axis represents the LOD score. The horizontal broken lines indicate the genome-wide threshold LOD score for significant $(P<0.05)$ and suggestive $(P<0.63)$ linkage, respectively.

3.2. Localization of Lipid QTL in $D B F_{2}$ and $B D F_{2}$ Males. Histograms showing the distributions of plasma $\mathrm{CHO}$ and TG concentrations in $300 \mathrm{~F}_{2}$ males (data from $150 \mathrm{BD}$ $\mathrm{F}_{2}$ and $150 \mathrm{DB} \mathrm{F}_{2}$ mice are combined) are presented in Figures 1 and 2. The distribution of $\mathrm{CHO}$ was bell-shaped (Figure 1(a)) but that of TG was slightly skewed (Figure 2(a)). Both traits were normalized after Box-Cox transformation (see Supplementary Figure 1 in the Supplementary Material available online at https://doi.org/10.1155/2017/3178204). For $\mathrm{CHO}$, the difference between $\mathrm{BD} \mathrm{F}_{2}(141 \pm 2 \mathrm{mg} / \mathrm{dL})$ and $\mathrm{DB}$ $\mathrm{F}_{2}(136 \pm 2 \mathrm{mg} / \mathrm{dL})$ males was not significant $(P>0.1)$. For $\mathrm{TG}$, the difference between $\mathrm{BD} \mathrm{F}_{2}(126 \pm 5 \mathrm{mg} / \mathrm{dL})$ and $\mathrm{DB} \mathrm{F}_{2}$ $(145 \pm 5 \mathrm{mg} / \mathrm{dL})$ males was significant $(P<0.002)$; therefore, cross direction (BD versus $\mathrm{DB})$ was included as a covariate in subsequent analyses.

Genome-wide LOD score plots obtained via single-QTL scans for plasma $\mathrm{CHO}$ and $\mathrm{TG}$ concentrations in $\mathrm{F}_{2}$ males are shown in Figures 1 and 2. For CHO (Figure 1(b), solid lines), we identified three significant QTL on Chr 1@80.5 cM (Choldq1), Chr 17@35.1cM (Choldq6), and Chr19@8.0cM (Choldq7) and three suggestive QTL on Chr 3@23.8 cM, Chr
5@59.8cM, and Chr 9@37.0 cM (Table 2). The suggestive QTL on Chr 5 coincided with Choldq4, which was previously identified as a significant QTL in C57BL/6J $\times$ DDD.Cg- $A^{y} \mathrm{~F}_{2}$ female populations [9]; therefore, we assigned the same gene symbol Choldq4 to this QTL. For TG (Figure 2(b), solid lines), we identified one significant QTL on Chr1@84.5 cM (Trigdq1) and four suggestive QTL on Chr 5@50.8 cM, Chr 12@47.0 cM, Chr 14@60.3cM, and Chr 15@53.9cM (Table 2). We also performed two-QTL scans to identify possible pairwise interactions between QTL but failed to identify significant interactions for both traits. Multiple-regression analyses indicated that the detected QTL explain 56.7 and $27.5 \%$ of the variations in plasma $\mathrm{CHO}$ and $\mathrm{TG}$ concentrations, respectively (Table 3).

There is a significant correlation between body weight and plasma lipid concentrations. That is, based on Spearman's rank correlation coefficient, the correlation between body weight and $\mathrm{CHO}$ concentration was 0.4855 (Spearman's $\rho$, $P<0.0001$ ), the correlation between body weight and TG concentration was 0.4505 (Spearman's $\rho, P<0.0001$ ), and the correlation between $\mathrm{CHO}$ and TG concentration was 0.4874 


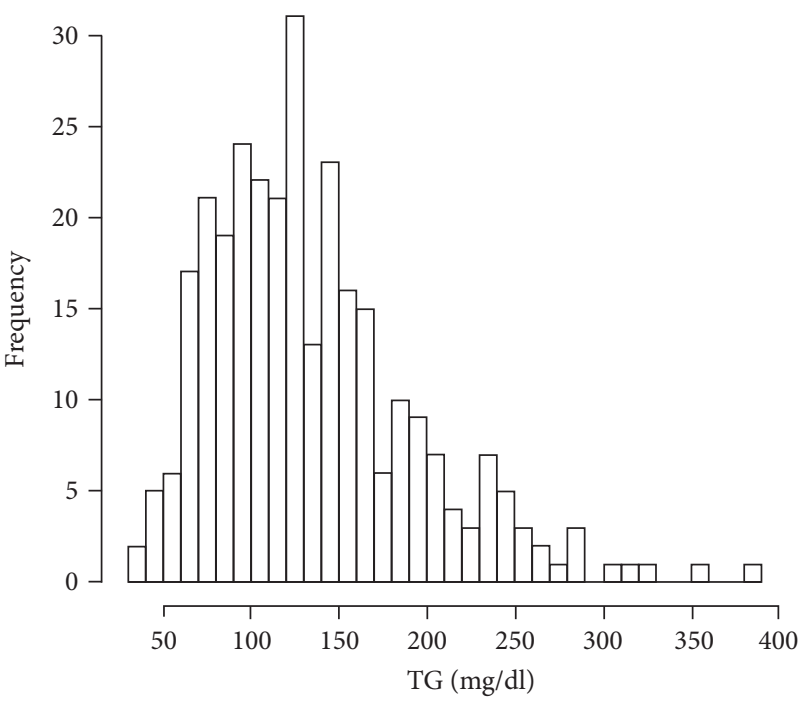

(a)

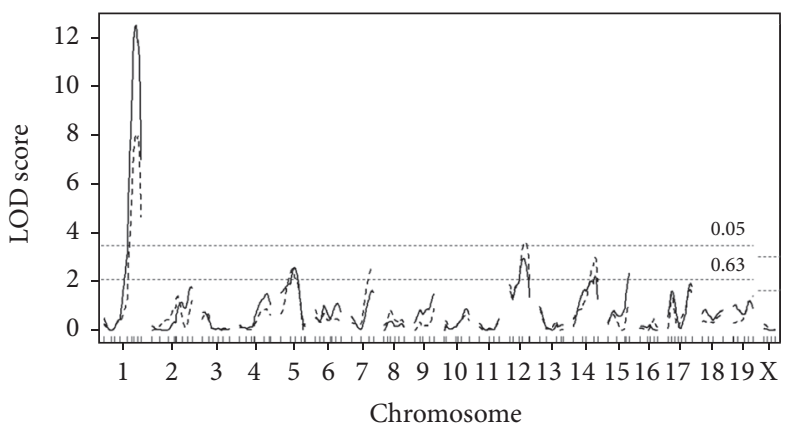

(b)

FIGURE 2: Genome-wide scan for plasma TG concentrations in $F_{2}$ male mice. (a) A histogram showing the distribution of plasma TG concentrations. (b) Genome-wide LOD score plot of single-QTL scans for plasma TG concentrations (solid lines: cross direction as an additive covariate; broken lines: cross direction and body weight as additive covariates). The $x$-axis represents the Chrs and microsatellite marker positions, and the $y$-axis represents the LOD score. The horizontal broken lines indicate the genome-wide threshold LOD score for significant $(P<0.05)$ and suggestive $(P<0.63)$ linkage, respectively.

TABLE 2: Significant and suggestive QTL identified by genome-wide scans of $\mathrm{F}_{2}$ males.

\begin{tabular}{|c|c|c|c|c|c|c|c|c|c|}
\hline \multirow{2}{*}{ Trait } & \multirow{2}{*}{$\mathrm{QTL}^{\mathrm{a}}$} & \multirow{2}{*}{$\mathrm{Chr}$} & \multirow{2}{*}{ Peak cM } & \multirow{2}{*}{$95 \% \mathrm{CI}^{\mathrm{b}}$} & \multirow{2}{*}{$\mathrm{LOD}^{\mathrm{c}}$} & \multirow{2}{*}{ Nearest marker } & \multirow{2}{*}{ High strain $^{\mathrm{d}} ;$ inheritance $^{\mathrm{e}}$} & \multicolumn{2}{|c|}{ Overlapping QTL } \\
\hline & & & & & & & & Name & Reference \\
\hline \multirow{6}{*}{$\mathrm{CHO}$} & Choldq1 & 1 & 80.5 & $77.5-85.5$ & 32.7 & D1Mit356 & DDD, Add & \multirow{2}{*}{ Cq2, Cq6, Hdlq20 } & \multirow{2}{*}[6,8,12]{} \\
\hline & \multirow{3}{*}{ Choldq4 } & 3 & 23.8 & $10.8-56.8$ & 2.3 & D3Mit25 & B6 & & \\
\hline & & 5 & 59.8 & $17.8-75.8$ & 2.9 & D5Mit239 & DDD & \multirow{2}{*}{ Hdlq1, Hdlq8 } & \multirow{2}{*}{ [13] } \\
\hline & & 9 & 37.0 & $12.0-59.6$ & 2.2 & D9Mit207 & DDD & & \\
\hline & Choldq6 & 17 & 35.1 & $17.1-51.1$ & 3.4 & D17Mit152 & B6, Add & Hdlq29 & {$[12]$} \\
\hline & Choldq7 & 19 & 8.0 & $3.0-19.0$ & 5.0 & D19Mit68 & DDD, Rec & Hdlq32, Hdlq48 & {$[12,14]$} \\
\hline \multirow{5}{*}{ TG } & \multirow[t]{2}{*}{ Trigdq1 } & 1 & 84.5 & $77.5-93.5$ & 12.5 & D1Mit356 & DDD, Dom & \multirow{2}{*}{$\operatorname{Tgq3}$} & \multirow{2}{*}[8]{} \\
\hline & & 5 & 50.8 & $17.8-66.8$ & 2.6 & D5Mit239 & DDD & & \\
\hline & \multirow[t]{3}{*}{ Trigdq2 } & 12 & 47.0 & $13.0-62.0$ & 2.9 & D12Mit259 & B6 & \multirow{3}{*}{$\operatorname{Tgq} 23$} & \multirow{3}{*}[15]{} \\
\hline & & 14 & 60.3 & $15.3-66.1$ & 2.3 & D14Mit165 & DDD & & \\
\hline & & 15 & 53.9 & $40.8-53.9$ & 2.4 & D15Mit193 & DDD & & \\
\hline
\end{tabular}

QTL, quantitative trait loci; CI, confidence interval; LOD, logarithm of the odds.

Cross direction was included as an additive covariate in all analyses.

${ }^{a}$ QTL symbols, Choldq4 and Trigdq2 were assigned to suggestive QTL on Chrs 5 and 12, respectively, because they were identified as significant QTL if the body weight was included as an additive covariate. Choldq4 was identified as significant QTL in our previous study in female mice [9].

${ }^{\mathrm{b}} 95 \%$ CI was defined by a 1.5-LOD decrease.

${ }^{c}$ LOD scores for significant QTL are indicated in bold. For CHO, the threshold LOD scores for significant and suggestive QTL were 3.4 and 2.1, respectively, for autosomes and 2.8 and 1.5, respectively, for Chr X. For TG, the threshold LOD scores for significant and suggestive QTL were 3.5 and 2.1, respectively, for autosomes and 2.8 and 1.5, respectively, for Chr X.

${ }^{\mathrm{d}}$ High strain-derived allele was associated with higher plasma lipids.

${ }^{\mathrm{e}}$ Mode of inheritance of high strain-derived allele. Dom, dominant; Add, additive; Rec, recessive.

(Spearman's $\rho, P<0.0001)$. Therefore, we next performed single-QTL scans by including body weight together with cross direction as additive covariates. As a result, the abovementioned suggestive QTL for $\mathrm{CHO}$ on $\mathrm{Chr} 5$ and suggestive QTL for TG on Chr 12 were identified as significant QTL (LOD score was 3.7 for both QTL) (Figures 1(b) and 2(b), broken lines). We named QTL on Chr 12 Trigdq2 (Table 2). We also performed two-QTL scans; however, there were again no significant pairwise interactions for both traits.

We next searched for possible QTL that interact with cross direction (BD versus $\mathrm{DB}$ ) by including cross direction as an interactive covariate. For $\mathrm{CHO}$ (but not TG), we 
TABLE 3: Multiple-regression analysis for plasma lipid concentrations.

\begin{tabular}{|c|c|c|c|c|}
\hline Plasma lipid & Chromosome $(\mathrm{cM})^{\mathrm{a}}$ & $\mathrm{df}^{\mathrm{b}}$ & Variance, $\%^{\mathrm{c}}$ & $F$ value \\
\hline \multirow{7}{*}{$\mathrm{CHO}$} & Chr1@80.5 & 2 & 34.1 & 113.0 \\
\hline & Chr3@23.8 & 2 & 2.0 & 6.6 \\
\hline & Chr5@59.8 & 2 & 3.3 & 11.0 \\
\hline & Chr9@37.0 & 2 & 4.0 & 13.3 \\
\hline & Chr17@35.1 & 2 & 4.7 & 15.5 \\
\hline & Chr19@8.0 & 2 & 3.1 & 10.1 \\
\hline & Total & 12 & 56.7 & \\
\hline \multirow{6}{*}{ TG } & Chr1@84.5 & 2 & 15.1 & 30.1 \\
\hline & Chr5@50.8 & 2 & 2.0 & 4.0 \\
\hline & Chr12@47.0 & 2 & 2.7 & 5.4 \\
\hline & Chr14@60.3 & 2 & 2.5 & 5.1 \\
\hline & Chr15@53.9 & 2 & 1.6 & 3.2 \\
\hline & Total & 10 & 27.5 & \\
\hline
\end{tabular}

Cross direction was also included as a covariate.

${ }^{\mathrm{a}} \mathrm{cM}$ position on the chromosome.

${ }^{\mathrm{b}}$ Degrees of freedom.

${ }^{\mathrm{c}}$ Percentage of the total $\mathrm{F}_{2}$ phenotypic variance associated with each marker.

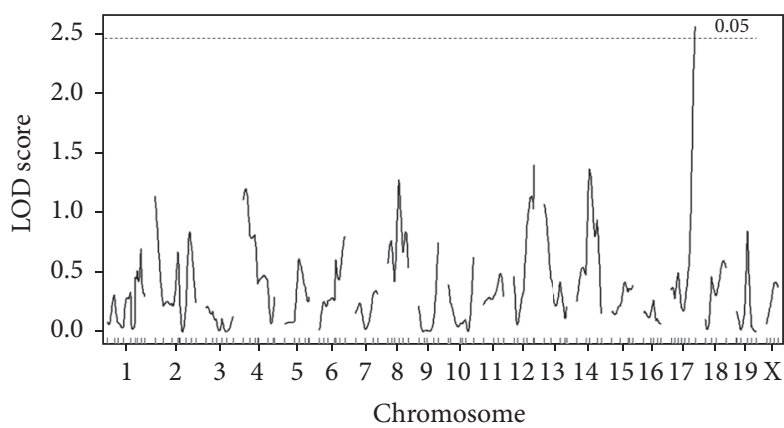

(a)

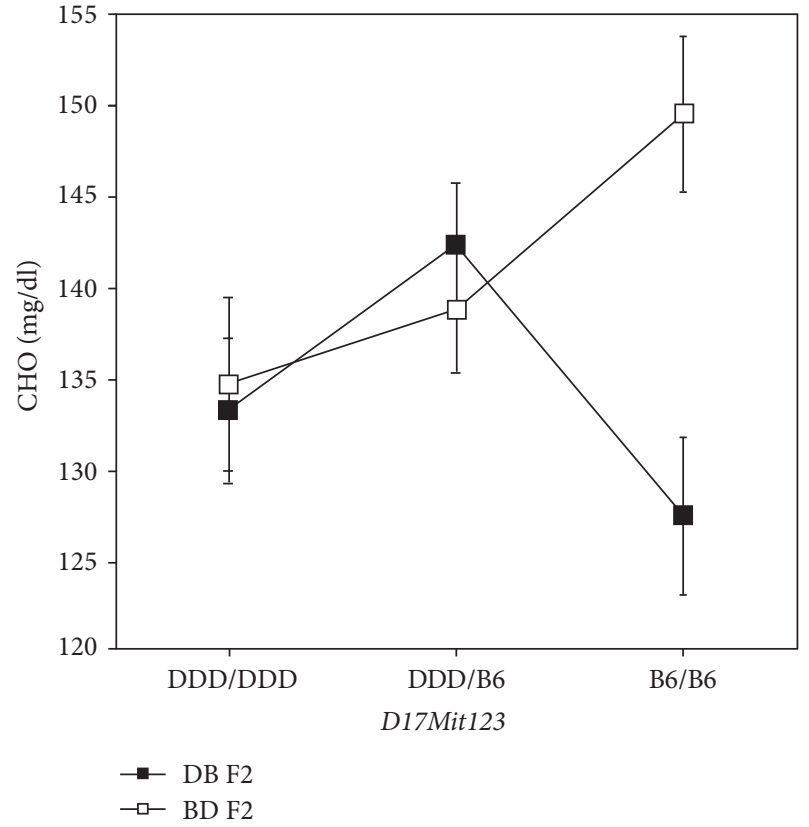

(b)

FIGURE 3: Genome-wide scan for QTL $\times$ cross direction interaction for plasma CHO concentrations. (a) Genome-wide LOD score plot. The $x$-axis represents the Chrs and microsatellite marker positions, and the $Y$-axis represents the LOD score. The genome-wide threshold LOD scores for a significant QTL $\times$ cross direction interaction were 2.4 for autosomes and 3.2 for $\mathrm{Chr} \mathrm{X}$, as indicated by horizontal broken line. (b) Allelic contributions of Choldq8, which interacts with cross direction. The $x$-axis shows the genotypes of $\mathrm{F}_{2}$ mice partitioned according to the nearest marker locus genotypes: homozygous DDD alleles are represented by DDD/DDD, homozygous B6 alleles are represented by $\mathrm{B} 6 / \mathrm{B} 6$, and heterozygous alleles are represented by DDD/B6. The $y$-axis shows the average $\mathrm{CHO}$ concentrations, and the error bars are SEM.

identified significant QTL that interacted with cross direction on Chr 17@60.7cM with LOD score 2.6 (threshold LOD score for significant QTL $\times$ covariate interaction was 2.4) (Figure 3(a)). Although the 95\% confidence interval (CI) of this QTL (50.1-60.7 cM) slightly overlapped with that of Choldq6 (17.1-51.1 cM), we assigned a new gene symbol, Choldq8, to this QTL, because the peak positions of the two QTL rather differed $(60.7 \mathrm{cM}$ versus $35.1 \mathrm{cM})$. The allele effects of this QTL in DB $F_{2}$ and $\mathrm{BD} \mathrm{F}_{2}$ mice are shown in Figure 3(b) (although the $\mathrm{CHO}$ concentrations are shown in 
TABLE 4: Significant and suggestive QTL when D1Mit356 was included as an additive covariate.

\begin{tabular}{|c|c|c|c|c|c|c|c|c|c|}
\hline \multirow{2}{*}{ Trait } & \multirow{2}{*}{$\mathrm{QTL}^{\mathrm{a}}$} & \multirow{2}{*}{ Chr } & \multirow{2}{*}{ Peak cM } & \multirow{2}{*}{$95 \% \mathrm{CI}^{\mathrm{b}}$} & \multirow{2}{*}{$\operatorname{LOD}^{c}$} & \multirow{2}{*}{ Nearest marker } & \multirow{2}{*}{ High strain $^{\mathrm{d}}$; inheritance ${ }^{\mathrm{e}}$} & \multicolumn{2}{|c|}{ Overlapping QTL } \\
\hline & & & & & & & & Name & Reference \\
\hline \multirow{7}{*}{$\mathrm{CHO}$} & Choldq9 & 3 & 19.8 & $10.8-35.8$ & 4.2 & D3Mit25 & B6, Add & $\mathrm{Cq3}$ & {$[6]$} \\
\hline & Choldq10 & 4 & 23.1 & $9.1-37.1$ & 3.6 & D4Mit286 & B6, Add & Hdlq10 & {$[16]$} \\
\hline & Choldq4 & 5 & 59.8 & $17.8-71.8$ & 3.7 & D5Mit239 & DDD, Rec & \multirow{2}{*}{$H d l q 1, H d l q 8$} & \multirow{2}{*}[13]{} \\
\hline & Choldq2 & 8 & 39.0 & $16.5-51.5$ & 2.8 & D8Mit263 & B6 & & \\
\hline & Choldq11 & 9 & 37.0 & $12.0-59.0$ & 4.7 & D9Mit207 & DDD, Add & Cq4, Cq5, Hdlq17 & {$[6,7,17]$} \\
\hline & Choldq6 & 17 & 37.1 & $15.1-50.1$ & 5.0 & D17Mit152 & B6, Rec & \multirow{2}{*}{ Hdlq29 } & \multirow{2}{*}[12]{} \\
\hline & Choldq7 & 19 & 5.0 & $3.0-19.0$ & 3.7 & D19Mit68 & DDD & & \\
\hline
\end{tabular}

QTL, quantitative trait loci; CI, confidence interval; LOD, logarithm of the odds.

Cross direction was also included as an additive covariate.

${ }^{a}$ QTL symbols were assigned if they were significant or if they were suggestive but were identified as a significant QTL at least once previously in different genetic crosses.

b $95 \%$ CI was defined by a 1.5-LOD decrease.

${ }^{\mathrm{c}}$ LOD scores for significant QTL are indicated in bold. The threshold LOD scores for significant and suggestive QTL were 3.4 and 2.1, respectively, for autosomes and 2.8 and 1.5 , respectively, for Chr X.

${ }^{\mathrm{d}}$ High strain-derived allele was associated with higher plasma lipids.

${ }^{\mathrm{e}}$ Mode of inheritance of high strain-derived allele. Add, additive; Rec, recessive.

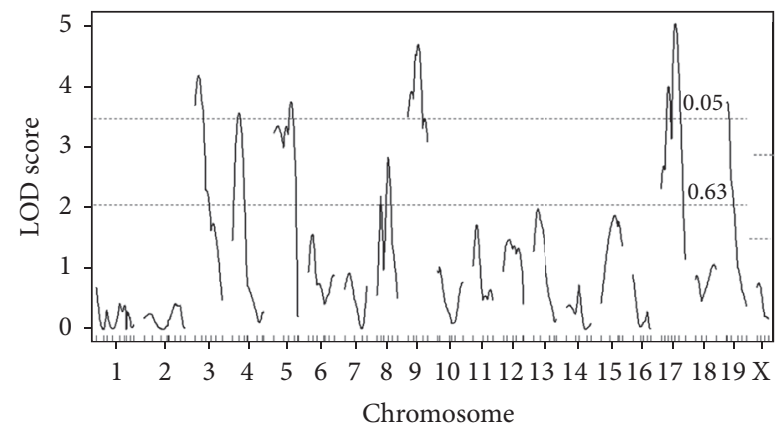

FIGURE 4: Genome-wide LOD score plot for CHO concentrations by composite interval mapping. The $x$-axis represents the $\mathrm{Chr}$ and microsatellite marker position, and the $y$-axis represents the LOD score. The horizontal broken lines indicate the genome-wide threshold LOD score for significant $(P<0.05)$ and suggestive $(P<$ $0.63)$ linkage, respectively.

$\mathrm{mg} / \mathrm{dL}$, statistical analyses were done on transformed data; the allele effects of this QTL on transformed data are shown in Supplementary Figure 2).

3.3. Localization of Lipid QTL in $F_{2}$ Males by Composite Interval Mapping. Because of the prominent phenotypic effect exerted by the Chr 1 QTL on both traits, we performed composite interval mapping by including the nearest marker for Choldq1/Trigdq1, that is, D1Mit356, and cross direction as additive covariates. Consequently, we identified six significant QTL for CHO, that is, Chr 3@19.8cM (Choldq9), Chr 4@23.1cM (Choldq10), Chr 5@59.8cM (Choldq4), Chr 9@37.0 cM (Choldq11), Chr 17@37.1cM (Choldq6), and Chr 19@5.0 cM (Choldq7) (Figure 4 and Table 4). In contrast, we did not identify any significant QTL for TG.

3.4. Localization of Lipid QTL in Combined $F_{2}$ Mice. Finally, we combined the data from this study on males with the previously analyzed data on $\mathrm{B} 6 \times \mathrm{DDD} \cdot \mathrm{Cg}-A^{y} \mathrm{~F}_{2}$ females [9] and performed QTL mapping analysis. This analysis first aimed to increase the power of QTL mapping, as there was a possibility that we could identify additional and/or novel QTL and that the 95\% CI for QTL could be narrowed by increasing the sample size. Second, we sought to identify possible QTL $\times$ sex interactions, as there may be QTL that significantly interact with sex. Such QTL would explain the difference in the results of QTL mapping performed between males and females. Because we analyzed two types of $\mathrm{F}_{2}$ mice, $\mathrm{F}_{2} A^{y}$ and $\mathrm{F}_{2}$ non- $A^{y}$, in our previous study of $\mathrm{F}_{2}$ females, trait data were standardized to a mean of 0 and a variance of 1 within each group of $\mathrm{F}_{2}$ males, $\mathrm{F}_{2} A^{y}$ females, and $\mathrm{F}_{2}$ non- $A^{y}$ females, prior to analysis. As shown in Supplementary Figures $3 \mathrm{~A}$ and $3 \mathrm{~B}$, standardized $\mathrm{CHO}$ followed a normal distribution but standardized TG did not. When sex was included as an additive covariate, we identified one additional significant QTL on Chr11@61.4 cM (Choldq12) for $\mathrm{CHO}$ but none for TG (Table 5). We did not identify any additional significant QTL for TG by nonparametric interval mapping. Although the LOD scores for some QTL were increased, the 95\% CIs for QTL were not necessarily narrowed sufficiently (e.g., Choldq1 on Chr 1). By including sex as an interactive covariate, we next searched for possible QTL $\times$ sex interactions for $\mathrm{CHO}$. We identified one significant sex-interacting QTL on Chr 19@10.0 cM (LOD score: 3.6, 95\% CI: 3.0-26.0 cM) (Figure 5(a)). This QTL coincided with Choldq7, which was identified by the single-QTL scan; therefore, we did not assign a new gene symbol to this QTL. The allele effects of this QTL in females and males are shown in Figure 5(b). The DDD allele tended to be associated with decreased $\mathrm{CHO}$ concentrations in females, whereas the DDD allele was significantly associated with increased $\mathrm{CHO}$ concentrations in males (although the $\mathrm{CHO}$ concentrations are shown in $\mathrm{mg} / \mathrm{dL}$, statistical analyses were done on standardized data; the allele effects of this QTL on standardized data are shown in Supplementary Figure 4). 


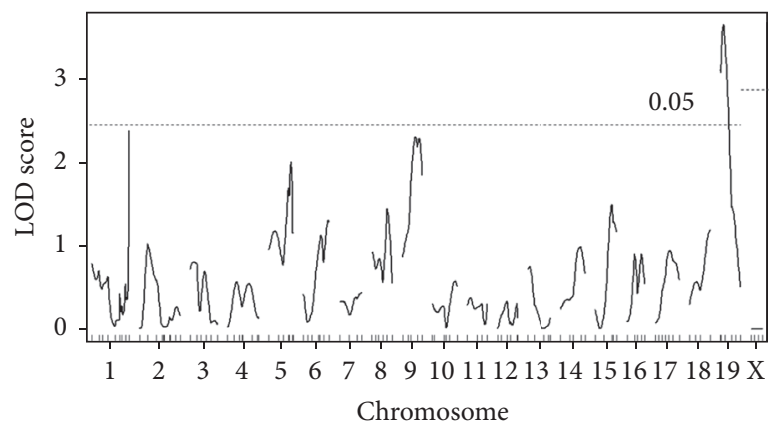

(a)

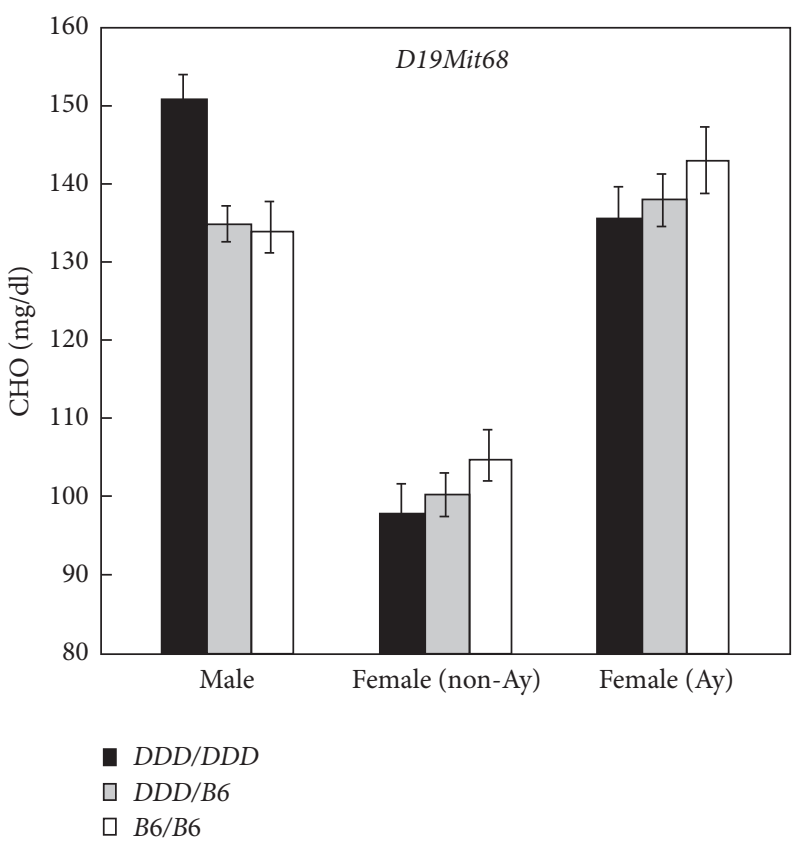

(b)

FIGURE 5: Genome-wide scan for QTL $\times$ sex interaction for plasma CHO concentrations. (a) Genome-wide LOD score plot. The $x$-axis represents the Chr and microsatellite marker position, and the $y$-axis represents the LOD score. The genome-wide threshold LOD scores for a significant QTL $\times$ sex interaction were 2.4 for autosomes and 2.9 for $\mathrm{Chr}$ X, as indicated by horizontal broken line. (b) Plasma CHO concentrations based on sex and the genotypes at D19Mit68. DDD/DDD denotes mice homozygous for the DDD allele, DDD/B6 denotes mice heterozygous for the DDD and B6 alleles, and B6/B6 denotes mice homozygous for the B6 allele. Error bars indicate SEM. In female mice, two kinds of $\mathrm{F}_{2}$ mice, that is, non- $A^{y}$ (lean) and $A^{y}$ (obese), according to the genotype at the agouti locus were analyzed [9].

TABLE 5: Significant and suggestive QTL for CHO identified by genome-wide scans of combined $\mathrm{F}_{2}$ mice $(n=598)$.

\begin{tabular}{|c|c|c|c|c|c|c|}
\hline $\mathrm{QTL}^{\mathrm{a}}$ & Chr & Peak cM & $95 \% \mathrm{CI}^{\mathrm{b}}$ & $\mathrm{LOD}^{\mathrm{c}}$ & Nearest marker & High strain $^{\mathrm{d}}$ \\
\hline Choldq1 & 1 & 79.2 & $77.5-82.5$ & 64.7 & Apoa2 & DDD \\
\hline Choldq9 & 3 & 41.8 & $12.8-62.8$ & 2.5 & D3Mit25 & B6 \\
\hline Choldq4 & 5 & 47.8 & $17.8-72.0$ & 2.1 & D5Mit239 & DDD \\
\hline Choldq2 & 8 & 21.2 & $14.5-53.5$ & 4.4 & D8Mit191 & B6 \\
\hline \multirow[t]{2}{*}{ Choldq12 } & 11 & 61.4 & $34.4-75.4$ & 3.5 & D11Mit124 & DDD \\
\hline & 12 & 60.6 & $23.0-62.2$ & 2.5 & D12Mit141 & B6 \\
\hline Choldq6 & 17 & 19.7 & $13.1-49.1$ & 3.3 & D17Mit176 & B6 \\
\hline
\end{tabular}

QTL, quantitative trait loci; CI, confidence interval; LOD, logarithm of the odds.

Sex was included as an additive covariate.

${ }^{a}$ QTL symbols were assigned if they were significant or if they were suggestive but were identified as significant QTL at least once previously in different genetic crosses [9].

${ }^{\mathrm{b}} 95 \%$ CI was defined by a 1.5-LOD decrease.

${ }^{c}$ LOD scores for significant QTL are indicated in bold. The threshold LOD scores for significant and suggestive QTL were 3.5 and 2.1 , respectively, for autosomes and 3.7 and 2.1, respectively, for $\mathrm{Chr} \mathrm{X}$.

${ }^{\mathrm{d}}$ High strain-derived allele was associated with higher plasma CHO.

3.5. Candidate Gene Search of Sex-Interacting QTL. We submitted the term "abnormal circulating cholesterol level" as a query to the MGI database (Mammalian Phenotype Browser), resulting in the retrieval of 1197 genotypes with 1905 annotations (MGI search was done on April 28, 2017). We consulted the MGI database (Genes and Markers Query Form) to determine the chromosomal localization of candidate genes. We performed whole-exome sequence analysis to identify nonsynonymous single-nucleotide variation
(nsSNV), frameshift, and nonsense mutations as well as insertion-deletion (indel) in the coding regions of candidate genes in DDD mice. Of note, we found an Asp94Gly aminoacid substitution in Acads (caused by c.281A $>\mathrm{G}$ at Chr 5: $115,113,143)$ in DDD mice; this had been proposed as the nsSNV underlying the HDL-CHO QTL on Chr 5 [22] (see Discussion). We inspected genes on Chr 19 in particular because only a few QTL for relevant traits have been mapped to this chromosome. We found five candidate genes located 
within the 95\% CI of Choldq7 (Lrp5, Pitpnm1, Naa40, Mark2, and $B s c l 2$ ) by MGI search but did not find any nsSNVs in any of these genes by whole-exome sequence analysis.

\section{Discussion}

This study identified a multifactorial basis for plasma lipid concentrations in $\mathrm{F}_{2}$ male mice generated by crosses between B6 and DDD inbred mice. We previously performed QTL mapping for body weight in the same $\mathrm{F}_{2}$ intercross population and identified four significant QTL on Chrs 1, 2, 5, and 17 [11]. Of these QTL, those on Chrs 1 and 5 coincided with QTL for CHO and TG concentrations, and that on Chr 17 coincided with QTL for $\mathrm{CHO}$ concentration on the basis of chromosomal localization (Table 2). Furthermore, the allele effect of each body weight QTL was in the same direction with that of lipid QTL. These results suggest a genetic link between body weight and plasma lipid concentrations. Indeed, we identified additional significant QTL, Choldq4 and Trigdq2, by including body weight as an additive covariate. These QTL may have indirect effect on plasma lipid concentrations by acting through body weight. Because dyslipidemia and obesity often coincide with cardiovascular disease, knowledge of the genetic factors common to body weight and plasma lipid concentrations will be crucial for our understanding of the disease [13].

There was a clear lineage effect on plasma lipid concentrations between $\mathrm{DB} \mathrm{F}_{1}$ and $\mathrm{BD} \mathrm{F}_{1}$ males; that is, $\mathrm{DB} \mathrm{F}_{1}$ mice had higher plasma lipids than $\mathrm{BD}_{1}$ mice. Based on the studies of Y-consomic mice, the lineage effect was not due to influence by Chr Y [23, 24]. Furthermore, because we observed the lineage effect in the comparison between $\mathrm{F}_{1}$ females, we concluded that the effect of Chr Y on plasma lipid concentrations is extremely small or nonexistent. Because Choldq8 (Chr17@60.7cM) interacts with cross direction, this QTL is involved in the observed lineage effect on $\mathrm{CHO}$ concentration. However, we cannot explain the lineage effect on TG concentration.

Most of the QTL identified in this study have coincidental QTL that had been reported by others $[3,4]$. Above all, Choldq1 on distal Chr 1 has numerous coincidental QTL for $\mathrm{CHO}$ and HDL-CHO concentrations. As observed for Choldq1, QTL identified in this region are known to have extremely large phenotypic effects $[6,8,9]$. The gene underlying these QTL is most likely Apoa2. When the crosses were composed of strains carrying different Apoa2 alleles (where one strain must have an Apoa $2^{b}$ allele), significant CHO QTL were invariably mapped to this chromosomal region [25]. Because DDD mice have the Apoa $2^{b}$ allele and B6 mice have the $A p o a 2^{a}$ allele, it is unsurprising that a significant $\mathrm{CHO}$ QTL was mapped to this region. Thus, Choldq1 is probably allelic with $C q 2, C q 6$, and $H d l q 20[6,8,12]$.

Choldq9 on Chr 3 was a coincidental QTL with Cq3, which was previously identified in $\mathrm{B} 6 \times \mathrm{KK} \cdot \mathrm{Cg}-A^{y} \mathrm{~F}_{2}$ mice [6]. Both Choldq9 and Cq3 might be allelic because the B6 allele was associated with increased $\mathrm{CHO}$ concentrations in the two studies.

Choldq4 on Chr 5 has at least two coincidental QTL for HDL-CHO, that is, Hdlq1 (125 Mb) and Hdlq8 (113 Mb) [22].
Su et al. [22] identified Scarb1 as the gene underlying Hdlq1 and Acads as the gene underlying Hdlq8. In particular, they demonstrated that the Asp94Gly amino-acid substitution in Acads was likely to alter the function of this protein because this change occurred in a highly conserved region. The Gly allele was associated with high HDL-CHO concentrations. DDD mice had the Gly allele, and B6 mice carried the Asp allele, suggesting that this was the nsSNV underlying Choldq4. Interestingly, we did not identify even a suggestive CHO QTL on Chr 5 in B6 $\times \mathrm{RR} \mathrm{F}_{2}$ mice [8], and both RR and B6 mice had the Asp allele (data not shown). Whereas Su et al. [22] reported they found no amino-acid changes in Scarbl, we identified one amino-acid change, Glu37Gln (caused by c. 109G>C at Chr 5: 125,304,335), in this protein. This aminoacid substitution was not identified in RR mice, in concordance with the absence of $\mathrm{CHO}$ QTL in $\mathrm{B} 6 \times \mathrm{RR} \mathrm{F}_{2}$ mice. Although the phenotypic effect of this amino-acid change is unclear, Scarb1 may also be regarded as a potential candidate gene underlying Choldq4.

Choldq11 on Chr 9 was a coincidental QTL with Cq4 and $C q 5$, which were previously identified in $\mathrm{B} 6 \times \mathrm{KK} . \mathrm{Cg}-A^{y} \mathrm{~F}_{2}$ mice [6] and in $\mathrm{KK} \times \mathrm{RR} \mathrm{F}_{2}$ mice [7], respectively. A proximal part of Chr 9 is known to have many genes relevant to lipid metabolism, including Apoa1, Apoc3, Apoa4, Apoa5, and Lipc, and multiple QTL including Cq4, Cq5, and Hdlq17 [17] have been reported. We previously hypothesized that the lipidassociated QTL on Chr 9 were most likely due to polymorphisms in Apoa4 [25]. We showed that a silent nucleotide change (c. $771 \mathrm{C}>\mathrm{T}$ ) in Apoa4 was well correlated with the incidence of the CHO QTL. However, we did not identify this nucleotide change in DDD mice. Furthermore, we did not identify any amino-acid changes in Apoal, Apoc3, Apoa5, and Lipc in DDD mice. Thus, we currently do not have any evidence that suggests that the above-mentioned genes are causative of Choldq11.

Based on their chromosomal localizations, two QTL have been reported as coincidental QTL with Choldq6 on Chr 17. One was identified in an interspecific cross between CAST/Ei and B6 (no gene symbols were assigned) [26] and the other was $H d l q 29$, which was identified in an intercross population between NZB/BINJ and B6 [12]. However, at variance with Choldq6, both QTL were identified only when the mice were fed on an atherogenic diet.

A relevant QTL mapped close to Choldq7 on Chr 19 is $H d l q 48$, which was identified in an $\mathrm{F}_{2}$ intercross between $\mathrm{B} 6$ and $\mathrm{A} / \mathrm{J}$ mice [14]. The peak position of Hdlq48 was $4 \mathrm{cM}$ with a $95 \%$ CI of $0-14 \mathrm{cM}$. However, at this QTL, the B6 allele was associated with higher HDL-CHO concentrations with an overdominant inheritance mode. These were apparently at variance with those of Choldq7, in which the B6 allele was associated with lower $\mathrm{CHO}$ concentrations with a dominant inheritance mode (Table 2). Another QTL, Hdlq32 (peak position of $26 \mathrm{cM}$ with a $95 \% \mathrm{CI}$ of $10-70 \mathrm{cM}$ ), was also located on Chr 19 [12]. Although the 95\% CI for Hdlq32 overlapped with that for Choldq7 (peak position of $7 \mathrm{cM}$ with a $95 \%$ CI of 3-20 cM), the peak position differed. Furthermore, there were substantial differences between the two QTL; that is, Hdlq32 was identified only when the mice were fed on a high-fat diet, and the phenotypic effect 
was observed only in females. Thus, there is no evidence supporting the possibility that both QTL are allelic. Thus, Choldq7 on Chr 19 was suggested to be a novel QTL.

Because of its chromosomal localization, Apoa2 appears to be the most suitable candidate gene underlying TG QTL (Trigdq1). However, we do not believe this to be the case for the following reasons. All three inbred strains, namely, $\mathrm{RR}$, DDD.Cg- $A^{y}$ (DDD), and KK.Cg- $A^{y}$ (KK), have the $A p o a 2^{b}$ allele. A significant TG QTL was identified in the Apoa2 region in $\mathrm{B} 6 \times \mathrm{RR} \mathrm{F}_{2}$ female mice [8], $\mathrm{B} 6 \times \mathrm{DDD} . \mathrm{Cg}-A^{y}$ (DDD) $\mathrm{F}_{2}$ female mice [9], and the present $\mathrm{F}_{2}$ male mice but was not identified in $\mathrm{B} 6 \times \mathrm{KK} . \mathrm{Cg}-A^{y}(\mathrm{KK}) \mathrm{F}_{2}$ mice [6]. Thus, it appears that RR and DDD mice carry a different Trigdq1 allele than KK mice, and we suspect that Apoa2 is not the gene underlying Trigdq1. Several reports support this hypothesis; for example, Ishimori et al. performed QTL mapping for $\mathrm{HDL}-\mathrm{CHO}$ and $\mathrm{TG}$ in $\mathrm{F}_{2}$ intercross populations between $\mathrm{B} 6$ and 129 S1/SvImJ inbred mice and identified significant HDLCHO QTL on distal Chr 1 but did not identify any TG QTL $[13,17]$. Su et al. [27] performed QTL mapping for HDL$\mathrm{CHO}$ and $\mathrm{TG}$ in $\mathrm{F}_{2}$ intercross populations between $\mathrm{B} 6$ and $\mathrm{C} 3 \mathrm{H} / \mathrm{HeJ}$ mice. They identified significant QTL for HDL$\mathrm{CHO}$ but not for TG. Thus, the gene underlying the TG QTL identified on distal Chr 1 in mouse intercross populations is unlikely to be Apoa2.

Although numerous QTL for plasma lipid concentrations have been reported, there are additional QTL that remain to be identified. QTL analyses using a new strain combination will identify additional QTL. Identification of coincidental QTL will further substantiate the candidate genes underlying previously reported QTL.

\section{Conflicts of Interest}

The authors declare that there are no conflicts of interest.

\section{Acknowledgments}

This study was supported in part by the NIAS (National Institute of Agrobiological Sciences) Strategic Research Fund.

\section{References}

[1] R. W. Hyman, S. Frank, C. H. Warden, A. Daluiski, R. Heller, and A. J. Lusis, "Quantitative trait locus analysis of susceptibility to diet-induced atherosclerosis in recombinant inbred mice," Biochemical Genetics, vol. 32, no. 11-12, pp. 397-407, 1994.

[2] D. A. Purcell-Huynh, A. Weinreb, L. W. Castellani, M. Mehrabian, M. H. Doolittle, and A. J. Lusis, "Genetic factors in lipoprotein metabolism: Analysis of a genetic cross between inbred mouse strains $\mathrm{NZB} / \mathrm{BINJ}$ and $\mathrm{SM} / \mathrm{J}$ using a complete linkage map approach," Journal of Clinical Investigation, vol. 96, no. 4, pp. 1845-1858, 1995.

[3] X. Wang and B. Paigen, "Genetics of variation in HDL cholesterol in humans and mice," Circulation Research, vol. 96, no. 1, pp. 27-42, 2005.

[4] X. Wang and B. Paigen, "Genome-wide search for new genes controlling plasma lipid concentrations in mice and humans," Current Opinion in Lipidology, vol. 16, no. 2, pp. 127-137, 2005.
[5] D. J. Bautz, K. W. Broman, and D. W. Threadgill, "Identification of a novel polymorphism in X-linked sterol-4-alphacarboxylate 3-dehydrogenase (Nsdhl) associated with reduced high-density lipoprotein cholesterol levels in i/LnJ mice," G3: Genes, Genomes, Genetics, vol. 3, no. 9, pp. 1819-1825, 2013.

[6] J.-I. Suto, S. Matsuura, H. Yamanaka, and K. Sekikawa, "Quantitative trait loci that regulate plasma lipid concentration in hereditary obese KK and KK- $A_{y}$ mice," Biochimica et Biophysica Acta - Molecular Basis of Disease, vol. 1453, no. 3, pp. 385-395, 1999.

[7] J.-I. Suto and K. Sekikawa, "Quantitative trait locus analysis of plasma cholesterol and triglyceride levels in KK $\times$ RR F2 mice," Biochemical Genetics, vol. 41, no. 9-10, pp. 325-341, 2003.

[8] J.-I. Suto, Y. Takahashi, and K. Sekikawa, "Quantitative trait locus analysis of plasma cholesterol and triglyceride levels in C57BL/6J × RR F 2 mice," Biochemical Genetics, vol. 42, no. 9-10, pp. 347-363, 2004.

[9] J.-I. Suto, "Quantitative Trait Loci that control plasma lipid levels in an F2 intercross between C57BL/6J and DDD.Cg-Ay inbred mouse strains," Journal of Veterinary Medical Science, vol. 74, no. 4, pp. 449-456, 2012.

[10] F. J. Charchar, L. D. S. Bloomer, T. A. Barnes et al., "Inheritance of coronary artery disease in men: An analysis of the role of the y chromosome," The Lancet, vol. 379, no. 9819, pp. 915-922, 2012.

[11] J. Suto and M. Kojima, "Quantitative trait loci that control body weight in DDD/Sgn and C57BL/6J inbred mice," Mammalian Genome, vol. 28, no. 1-2, pp. 13-19, 2017.

[12] R. Korstanje, R. Li, T. Howard et al., "Influence of sex and diet on quantitative trait loci for HDL cholesterol levels in an SM/J by NZB/BINJ intercross population," Journal of Lipid Research, vol. 45, no. 5, pp. 881-888, 2004.

[13] N. Ishimori, R. Li, P. M. Kelmenson et al., "Quantitative trait loci that determine plasma lipids and obesity in C57BL/6J and 129S1/SvImJ inbred mice," Journal of Lipid Research, vol. 45, no. 9, pp. 1624-1632, 2004.

[14] I. M. Stylianou, S.-W. Tsaih, K. DiPetrillo et al., "Complex genetic architecture revealed by analysis of high-density lipoprotein cholesterol in chromosome substitution strains and F2 crosses," Genetics, vol. 174, no. 2, pp. 999-1007, 2006.

[15] I. M. Stylianou, S. R. Langley, K. Walsh, Y. Chen, C. Revenu, and B. Paigen, "Differences in DBA/1J and DBA/2J reveal lipid QTL genes," Journal of Lipid Research, vol. 49, no. 11, pp. 2402-2413, 2008.

[16] M. A. Lyons, H. Wittenburg, R. Li et al., "Quantitative trait loci that determine lipoprotein cholesterol levels in DBA/2J and CAST/Ei inbred mice," Journal of Lipid Research, vol. 44, no. 5, pp. 953-967, 2003.

[17] N. Ishimori, R. Li, P. M. Kelmenson et al., "Quantitative trait loci analysis for plasma hdl-cholesterol concentrations and atherosclerosis susceptibility between inbred mouse strains C57BL/6J and 129S1/SvImJ," Arteriosclerosis, Thrombosis, and Vascular Biology, vol. 24, no. 1, pp. 161-166, 2004.

[18] K. W. Broman and Ś. Sen, A Guide to QTL Mapping with R/qtl, Springer, New York, NY, USA, 2009.

[19] K. W. Broman, H. Wu, Ś. Sen, and G. A. Churchill, "R/qtl: QTL mapping in experimental crosses," Bioinformatics, vol. 19, no. 7, pp. 889-890, 2003.

[20] E. Lander and L. Kruglyak, "Genetic dissection of complex traits: guidelines for interpreting and reporting linkage results," Nature Genetics, vol. 11, no. 3, pp. 241-247, 1995.

[21] Ś. Sen and G. A. Churchill, "A statistical framework for quantitative trait mapping," Genetics, vol. 159, no. 1, pp. 371-387, 2001. 
[22] Z. Su, M. S. Leduc, R. Korstanje, and B. Paigen, "Untangling HDL quantitative trait loci on mouse chromosome 5 and identifying Scarbl and Acads as the underlying genes," Journal of Lipid Research, vol. 51, no. 9, pp. 2706-2713, 2010.

[23] J.-I. Suto, "Metabolic consequence of congenital asplenia caused by the Dominant hemimelia mutation in mice," Journal of Veterinary Medical Science, vol. 71, no. 2, pp. 177-182, 2009.

[24] J.-I. Suto and K. Satou, "Effect of the y chromosome on plasma high-density lipoprotein-cholesterol levels in Y-chromosomeconsomic mouse strains," BMC Research Notes, vol. 7, no. 1, article 393, 2014.

[25] J.-I. Suto, "Apolipoprotein gene polymorphisms as cause of cholesterol QTLs in mice," Journal of Veterinary Medical Science, vol. 67, no. 6, pp. 583-589, 2005.

[26] M. Mehrabian, L. W. Castellani, P.-Z. Wen et al., "Genetic control of HDL levels and composition in an interspecific mouse cross (CAST/Ei × C57BL/6J)," Journal of Lipid Research, vol. 41, no. 12, pp. 1936-1946, 2000.

[27] Z. Su, N. Ishimori, Y. Chen et al., "Four additional mouse crosses improve the lipid QTL landscape and identify lipg as a QTL gene," Journal of Lipid Research, vol. 50, no. 10, pp. 2083-2094, 2009. 


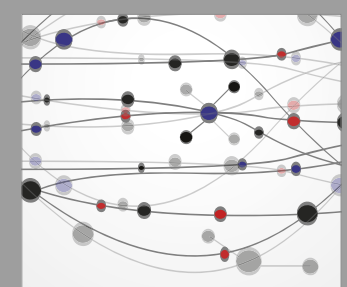

The Scientific World Journal


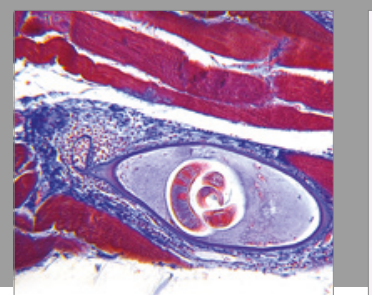

Gastroenterology Research and Practice
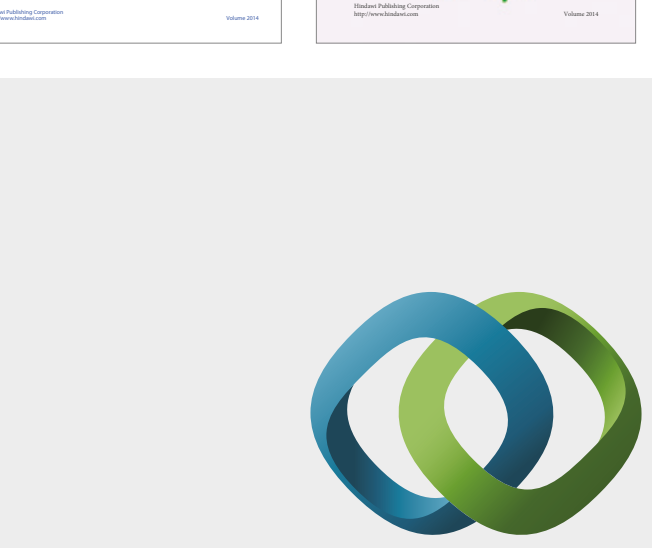

\section{Hindawi}

Submit your manuscripts at

https://www.hindawi.com
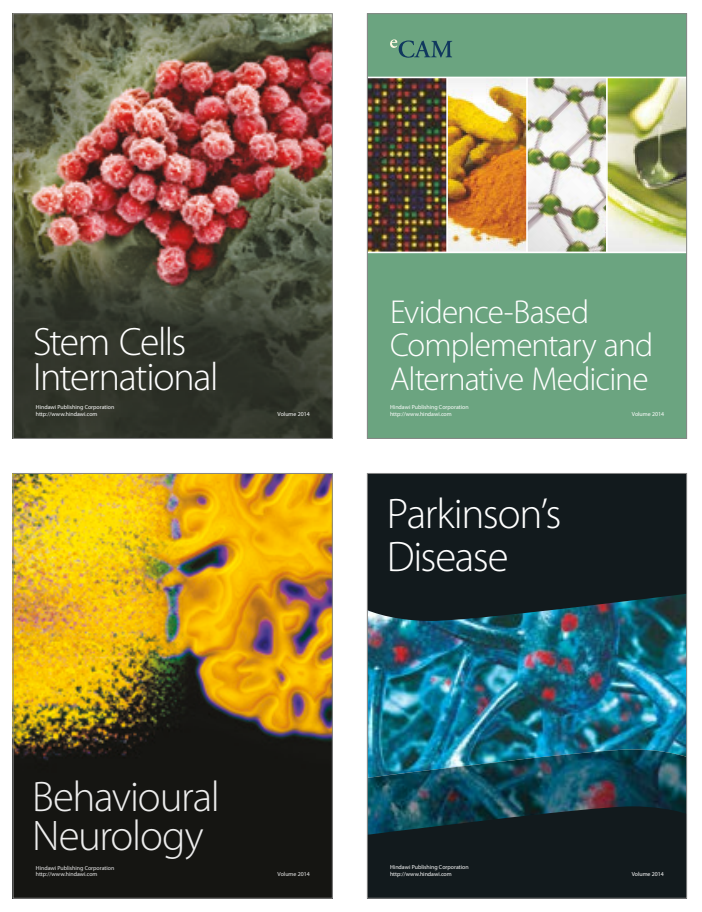
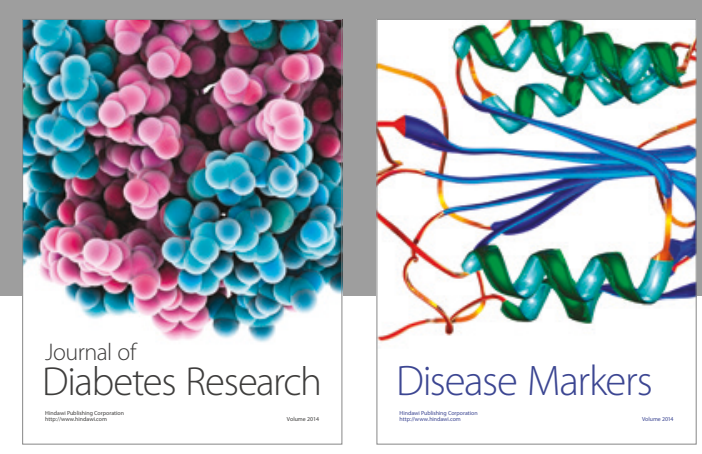

Disease Markers
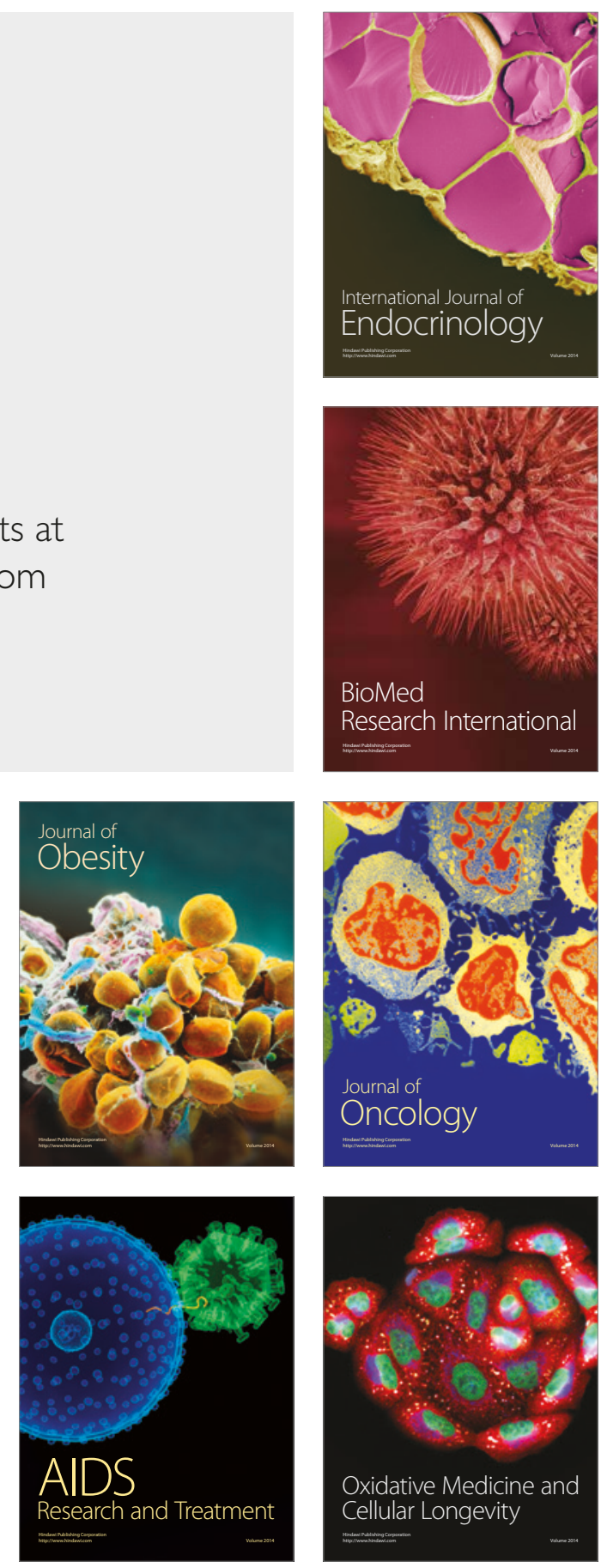